\title{
A Coarse-to-fine Framework for Automated Kidney and Kidney Tumor Segmentation from Volumetric CT Images
}

\author{
Huai Chen ${ }^{1}$, Xiyi $\mathrm{Wu}^{1}$, Yijie Huang ${ }^{1}$, and Lisheng Wang ${ }^{1}$ \\ Institute of Image Processing and Pattern Recognition, Department of Automation, \\ Shanghai Jiao Tong University, Shanghai, 200240, P. R. China.1swang@sjtu.edu.cn
}

\begin{abstract}
Automatic semantic segmentation of kidney and kidney tumor is a promising tool for the treatment of kidney cancer. Due to the wide variety in kidney and kidney tumor morphology, it is still a great challenge to complete accurate segmentation of kidney and kidney tumor. We propose a new framework based on our previous work accepted by MICCAI2019, which is a coarse-to-fine segmentation framework to realize accurate and fast segmentation of kidney and kidney tumor.
\end{abstract}

Keywords: Kidney · Kidney cancer · Segmentation · Coarse-to-fine.

\section{Introduction}

There are more than 400,000 new cases of kidney cancer each year [?], and surgery is its most common treatment [4]. Due to the wide variety in kidney and kidney tumor morphology, there is currently great interest in how tumor morphology relates to surgical outcomes, $[3,1]$ as well as in developing advanced surgical planning techniques [5]. Automatic semantic segmentation is a promising tool for these efforts, but morphological heterogeneity makes it a difficult problem.

The goal of this challenge is to accelerate the development of reliable kidney and kidney tumor semantic segmentation methodologies. We have produced ground truth semantic segmentations for arterial phase abdominal CT scans of 300 unique kidney cancer patients who underwent partial or radical nephrectomy at our institution. 210 of these have been released for model training and validation, and the remaining 90 will be held out for objective model evaluation

\section{Method}

The framework is shown as in fig. 1.Our proposed method is a coarse-to-fine segmentation framework, which use a lightweight 3D Unet to realize coarse segmenting to locate the ROI region of kidney. The we propose a dimension adaptation module (DAM) to bridge powerful 2D pre-trained network and 3D features 


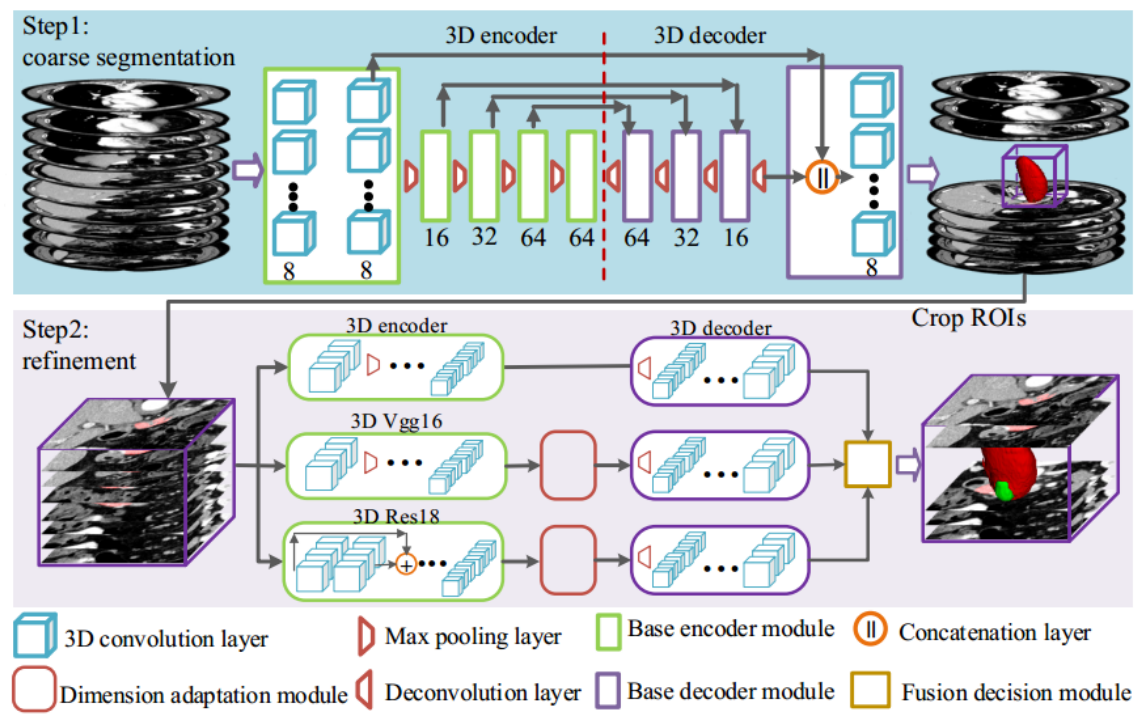

Fig. 1. An illustration of our framework. (a) 3D coarse segmentation network trained with bias-dice loss proposes ROIs with high recall rates. (b) Refinement network that uses DAMs to transform intra-features of pre-trained networks into 3D and the fusion decision module to combine multi-source features to make accurate predictions.

to realize accurate segmentation of ROIs. In the same time we propose a fusion decision module to effectively fuse multi-source features. Our method is accepted in MICCAI2019 [2].

The architecture of DAM is shown in fig.2, which captures interdependencies of adjacent slices to make final 3D predictions. The utilization of powerful pretrained 2D networks can greatly improve the segmentation of kidney and kidney tumor in 3D CT images.

\section{Training}

In order to accelerate the inference of to the large size of original CT images, we downsample images to $256 \times 256$ on $\mathrm{X}$ and $\mathrm{Y}$ axis in coarse segmentation stage. While, images with original resolution are utilized in fine segmenting stage. We clip (limit) values to reduce the complexity of data, we set the range of value as $[-100,300]$. And we normalize the image to $[-1,1]$.

For the training of all network, we use Adam with learning rate of 0.001 as the optimizer. We split 21 instances of training data as the validation data to choose the best model and the early-stopping strategy is utilized to alleviate overfitting. The max epoch number is 100 and the patient of early stopping is 5 . 


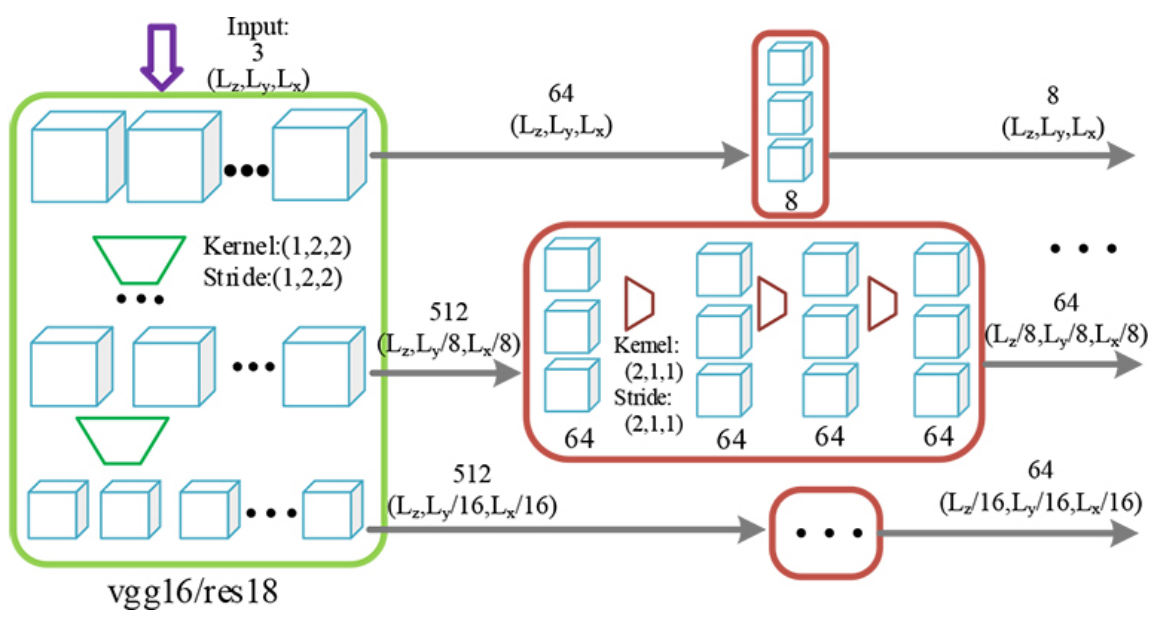

Fig. 2. The illustration of dimension adaptation module.

\section{Inference}

We firstly resize images to $256 \times 256$ on $\mathrm{X}$ and $\mathrm{Y}$ axis. Then, we feed them into the coarse segmentation network to obtain coarse results. Regions with first two large areas, with more than 4000 pixels, will be selected as the ROIs. The ROIs will be fed into the fine segmentation network to obtain accurate result.

\section{$5 \quad$ Results}
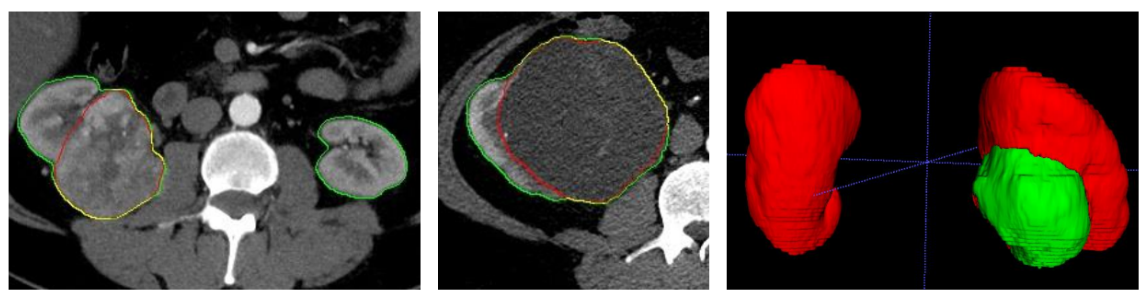

Fig. 3. Some prediction results in $2 \mathrm{D}$ and $3 \mathrm{D}$ images.

Some segmentation results of kidney and kidney tumor is shown in fig.5. The composite Dice in test data is 0.8587 , and Dice for kidney and kidney tumor are respectively 0.9655 and 0.7520 . 


\section{References}

1. Ficarra, V., Novara, G., Secco, S., Macchi, V., Porzionato, A., De Caro, R., Artibani, W.: Preoperative aspects and dimensions used for an anatomical (padua) classification of renal tumours in patients who are candidates for nephron-sparing surgery. European urology 56(5), 786-793 (2009)

2. Huai Chen, Xiuying Wang, Y.H.X.W.Y.Y., Wang, L.: Harnessing 2 d networks and $3 \mathrm{~d}$ features for automated pancreas segmentation from volumetric ct images. In: International Conference on Medical Image Computing and Computer-Assisted Intervention. Springer (2019)

3. Kutikov, A., Uzzo, R.G.: The renal nephrometry score: a comprehensive standardized system for quantitating renal tumor size, location and depth. The Journal of urology 182(3), 844-853 (2009)

4. Siegel, R., DeSantis, C., Virgo, K., Stein, K., Mariotto, A., Smith, T., Cooper, D., Gansler, T., Lerro, C., Fedewa, S., et al.: Cancer treatment and survivorship statistics, 2012. CA: a cancer journal for clinicians 62(4), 220-241 (2012)

5. Taha, A., Lo, P., Li, J., Zhao, T.: Kid-net: convolution networks for kidney vessels segmentation from ct-volumes. In: International Conference on Medical Image Computing and Computer-Assisted Intervention. pp. 463-471. Springer (2018) 